\title{
ORIGINAL ARTICLE \\ Quantitative genetics of immunity and life history under different photoperiods
}

\begin{abstract}
K Hammerschmidt ${ }^{1}$, P Deines ${ }^{1,3}$, AJ Wilson ${ }^{2}$ and J Rolff ${ }^{1}$
Insects with complex life-cycles should optimize age and size at maturity during larval development. When inhabiting seasonal environments, organisms have limited reproductive periods and face fundamental decisions: individuals that reach maturity late in season have to either reproduce at a small size or increase their growth rates. Increasing growth rates is costly in insects because of higher juvenile mortality, decreased adult survival or increased susceptibility to parasitism by bacteria and viruses via compromised immune function. Environmental changes such as seasonality can also alter the quantitative genetic architecture. Here, we explore the quantitative genetics of life history and immunity traits under two experimentally induced seasonal environments in the cricket Gryllus bimaculatus. Seasonality affected the life history but not the immune phenotypes. Individuals under decreasing day length developed slower and grew to a bigger size. We found ample additive genetic variance and heritability for components of immunity (haemocyte densities, proPhenoloxidase activity, resistance against Serratia marcescens), and for the life history traits, age and size at maturity. Despite genetic covariance among traits, the structure of G was inconsistent with genetically based trade-off between life history and immune traits (for example, a strong positive genetic correlation between growth rate and haemocyte density was estimated). However, conditional evolvabilities support the idea that genetic covariance structure limits the capacity of individual traits to evolve independently. We found no evidence for $\mathrm{G} \times \mathrm{E}$ interactions arising from the experimentally induced seasonality.
\end{abstract}

Heredity (2012) 108, 569-576; doi:10.1038/hdy.2011.125; published online 21 December 2011

Keywords: time constraints; trade-off; full-sib/half-sib design

\section{INTRODUCTION}

In animals with complex life-cycles, where individuals grow as juveniles only, life history theory predicts age and size at maturity to be optimized during larval development (Roff, 1992; Stearns, 1992). Here, the trade-off between large size and long development time should be particularly pronounced (Roff, 1980). For organisms inhabiting seasonal environments, reproductive periods are restricted (Werner, 1986; Ludwig and Rowe, 1990; Rowe and Ludwig, 1991). Thus, not only size, but also the time to attain a certain size is an important factor in organisms with time-constrained life histories (Rowe and Ludwig, 1991). Individuals that reach maturity late in the season have to either reproduce at a smaller size or increase their growth rates, leading to a strong selection pressure on growth rate. However, species inhabiting seasonal environments show high variance in their growth rates (Semlitsch, 1993; Bradshaw and Holzapfel, 1996) and one proposal is that variation might arise from adaptive plastic responses to variable environmental conditions (Abrams et al., 1996; Gotthard, 2001). Several studies have found costs and trade-offs associated with high growth rates in insects, with costs manifesting as higher juvenile mortality, decreased adult survival (Chippindale et al., 1994) or increased susceptibility to parasitism by bacteria and viruses (Sharpe and Detroy, 1979).

These costs associated with high growth rates could potentially serve as one explanation for the observation that size and age at maturity sometimes explain only a small amount of variation in adult fitness (De Block and Stoks, 2005). Recent work suggests that part of the variation in fitness can be explained by changes in physiological traits (Zera and Harshman, 2001; Rolff et al., 2004), such as immunity (Rolff and Siva-Jothy, 2003; Schmid-Hempel, 2003). For example, changes in photoperiod alter growth trajectories and can decouple life history and physiological traits (Rolff et al., 2004). Physiological parameters can be independent of size and age at maturity and thus may serve as independent targets of selection (Plaistow et al., 2004; Rolff et al., 2004). For example, damselflies developing under time constraints show reduced activity of phenoloxidase (PO), an important component of the insect immune system, but not changes in mass at emergence (Rolff et al., 2004). The notion that physiological traits and life history traits serve as independent targets of selection depends on the potential for unconstrained evolvabilities of these traits, yet quantitative genetic studies found evidence for greatly reduced evolvabilities using conditional estimates (Rolff et al., 2005).

Independent of studies elucidating the impact of seasonality on immune function and life histories (see above), data are also accumulating suggesting a genetic-based trade-off between life history traits and immune function (Coltman et al., 2001; Cotter et al., 2004; Rolff et al., 2005). Here, we combine experimental approaches manipulating photoperiod with a quantitative genetic analysis and ask, given that seasonal environments are very common, does envir-

${ }^{1}$ Department of Animal and Plant Sciences, The University of Sheffield, Sheffield, UK and ${ }^{2}$ Institute of Evolutionary Biology, School of Biological Sciences, University of Edinburgh, Edinburgh, UK

Correspondence: Dr K Hammerschmidt. Current address: NZ Institute for Advanced Study, Massey University, Private Bag 102904, Auckland, New Zealand.

E-mail: k.hammerschmidt@massey.ac.nz

${ }^{3}$ Current address: Institute of Natural Sciences, Massey University, Private Bag 102904, Auckland, New Zealand

Received 25 May 2011; revised 14 October 2011; accepted 11 November 2011; published online 21 December 2011 
onmental change alter the underlying genetic architecture of life history and immune function traits?

We use the cricket Gryllus bimaculatus, a hemimetabolous insect, living in a seasonal environment to experimentally investigate whether time constraints during insect larval development influence physiological traits in adults, specifically different components of the immune system. To ensure that the measured components of the immune system translate into an animal's ability to mount an effective immune response against pathogens (Adamo, 2004), we subsequently exposed the crickets to the entomopathogen Serratia marcescens. Choosing an insect as model system, allows growth patterns to be influenced by manipulating photoperiod without the confounding effects of other cues such as diet or temperature (Rolff et al., 2004).

We combine experimental manipulation of day length, using the natural ranges where $G$. bimaculatus occurs, with a full-sib/half-sib quantitative genetic approach, to investigate the following hypotheses: (i) Do life history and immune traits show phenotypic plasticity in response to the photoperiod treatment? We predict that individuals kept under late photoperiod conditions, that is, decreasing day length, should on average grow faster at the expense of investment into size and immunity compared with the ones raised under early photoperiod conditions. We then (ii) examine the quantitative genetic architecture of life history and immune traits, their heritability and evolvability, and test whether trade-offs exist between immune and life history traits at a genetic level. We then explore whether (iii) the genetic and environmental (that is, plasticity) processes interact such that the genetic architecture is environment dependent. Finally, we investigate the autonomy of the individual traits (iv), that is, the ability of any one trait to evolve under selection, if all other traits are under stabilizing selection (Hansen and Houle, 2008).

\section{MATERIALS AND METHODS}

\section{Design of experiment}

We used a full-sib/half-sib breeding design in which each of 50 male G. bimaculatus (sires) were singly mated to 5 females (dams) after the onset of sexual maturity. The male was removed once the sperm container, the spermatophore, was attached to the female and at 4 days females were provided with plastic vessels filled with moist humus for oviposition. After a further 2 days, the vessels were removed with nymphs hatching 3 weeks later. All animals and oviposition vessels were kept under constant temperature and photoperiod conditions. Nymphs produced from each mating were counted and paternal half-sibships were then included in the study provided that $\geqslant 80$ viable offspring were produced with three of the five dams. This was the case for 28 sires $\times 3$ dams (Figure 1 ). Nymphs from each sire $\times$ dam combination were

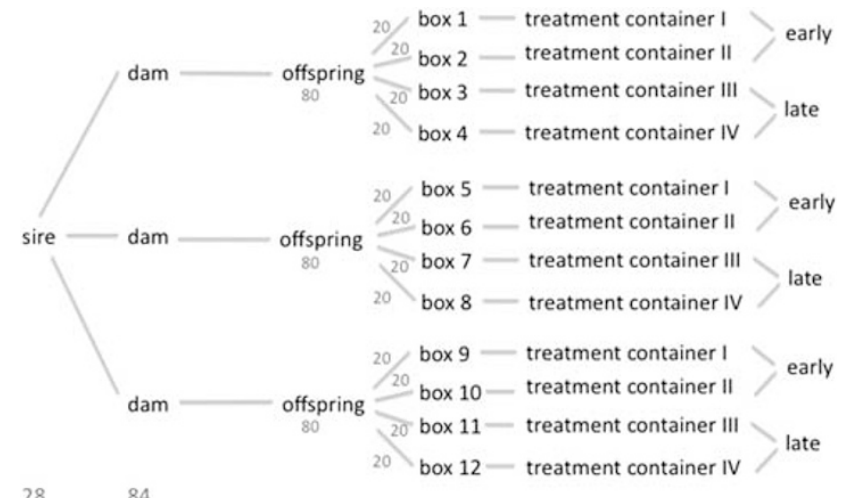

Figure 1 Schematic diagram of the experimental design. Shown is the example for one sire bred to three dams. Numbers in grey represent the sample size at the beginning of the photoperiod treatment. housed in plastic boxes containing groups of 20 individuals ( 4 boxes per cross, 84 crosses, total $N$ at the start of the experiment=6720), from which 1104 crickets developed into adults.

At the start of the experiment, these boxes were distributed over 16 treatment containers so that one set of boxes per sire (with the three dams, offspring of each mating were kept in an individual box) was in the same treatment container. The experiment was set up so that four of the 16 treatment containers contained the same sire $\times$ dam combinations. In half of the 16 treatment containers, the photoperiod was $12: 12 \mathrm{~h}$ (light:dark) to simulate hatching in early spring ('early'), in the other half it was $16: 8 \mathrm{~h}$ (light:dark) to simulate hatching in early summer ('late'). The temperature was kept constant at $26{ }^{\circ} \mathrm{C}$. Throughout the experiment, the photoperiods were adjusted every 7 days to simulate the natural process of the light cycle (Stoks et al., 2006), so that after 9 weeks, the light:dark ratio in the spring treatment was 16:8 h (light: dark), and in the summer treatment it was 12:12 h (light:dark). Since our pilot study indicated that crickets at this temperature need approximately 2 months to become adult, this set up ensured that the individuals experienced the same amount of light and dark during the experiment and differed only in when during their development they received it. Within every treatment, there were two replicated containers, containing the same sire $\times$ dam combinations. Every second day, the crickets were fed, until the first crickets became adult. From then onwards, the boxes were checked daily, to collect the freshly moulted adults, which were kept singly. The time to reach adulthood was recorded.

\section{Life pathogen infection}

Three to five days after each individual cricket reached adulthood, haemolymph was taken (see below) and animals were injected with a $\mathrm{LD}_{50}$ dose of Serratia marcescens through the pronotal membrane (cell concentration: $2.5 . \times 10^{6}$ per $5 \mu$ l Ringer; dose determined in a pre-experiment). $S$. marcescens is known to cause opportunistic infections in a wide range of insect species (Krieg, 1987). Cricket survival was monitored daily for the next 10 days post infection. Subsequently, all crickets were individually frozen at $-20^{\circ} \mathrm{C}$ so that sex and size (pronotum width, which is a good indicator for body size; Rolff, unpublished data) could be determined. So far, only a few studies that have investigated the relationship between PO activity and resistance to pathogens have found a significant correlation (Nigam et al., 1997; Siva-Jothy, 2000), whereas others found none (Adamo, 2004). By exposing the crickets to live pathogens, it was ensured that the measured components of the immune system could be linked to adult survival via susceptibility to pathogens.

\section{Haemolymph collection and counts}

Before haemolymph collection, individuals were cooled on ice for $\mathrm{ca} .15 \mathrm{~min}$. Haemolymph was then taken by puncturing the membrane below the pronotum (dorsal) with a sterile injection needle after the pronotum was cleaned with $70 \%$ ethanol. The sample was split into two parts: $5 \mu \mathrm{l}$ was directly frozen at $-80^{\circ} \mathrm{C}$ for proPO and lysozyme assays (see below), and $2 \mu \mathrm{l}$ was added to $48 \mu \mathrm{l}$ of phosphate-buffered saline (PBS) for haemocyte counts. The haemocyte-PBS mix was pipetted into a well of a multiwell microscope slide. Haemocytes were left to settle for a minimum of an hour in a humidified container, before $20 \mu \mathrm{l}$ of DAPI (4',6-diamidino-2-phenylindole; $1 \mu \mathrm{l}$ DAPI in $1 \mathrm{ml}$ PBS) was added and incubated for $5 \mathrm{~min}$. Then, it was carefully washed with PBS until clear. In the last washing step, $60 \mu \mathrm{l}$ of liquid were removed and a cover slip was placed on each slide. For each well, three pictures of randomly chosen (non-overlapping) areas were taken with a digital camera mounted on a microscope. Most haemocytes were counted with the image analysis programme Image Pro Plus 5.1 (MediaCybernatics, Bethesda, MD, USA) with the 'automatic bright object function' and marked on the picture. Cells that could not be automatically counted (because they were not bright enough or too dense) were not marked and were manually counted. This was done after the experiment had finished, and blind to treatment and sire $\times$ dam combination. For each cricket, the mean of the three haemocyte counts was used.

\section{ProPO assay}

For each individual, the haemolymph concentrations of the proenzyme proPO were measured following the protocol by Jacot et al. (2005). ProPO is the inactive form in which the majority of PO is stored. To be able to measure 
proPO, it needs to be activated. Here, we used chymotrypsin, one of several compounds known to activate proPO in vitro. To activate all proPO, it is essential to provide enough chymotrypsin; for G. bimaculatus we found $5 \mathrm{mg} \mathrm{ml}^{-1}$ of chymotrypsin to be the ideal concentration.

In the PO assay, the conversion of L-dopa into dopachrome is measured spectrographically (Barnes and Siva-Jothy, 2000; Gillespie et al., 2000). As for many other enzymes, the kinetic behaviour of insect $\mathrm{PO}$ can be described by the Michaelis-Menten equation (as exemplified by Thompson et al., 2002). To be able to measure the $V_{\max }$ (the maximum velocity of the enzyme reaction), the substrate L-dopa needs to be at its saturation concentration, which is speciesspecific. Following Henderson (1992) and testing 6 L-dopa concentrations, the saturated concentration was found to be $18 \mathrm{~mm} \mathrm{L-dopa} \mathrm{for} \mathrm{G.} \mathrm{bimaculatus.}$

For the assay, cricket haemolymph was carefully thawed on ice, centrifuged at 14000 r.p.m. for $4 \mathrm{~min}$ and $1 \mu \mathrm{l}$ sample taken. The protocol we use for measuring proPO is: mix $93 \mu \mathrm{H}_{2} \mathrm{O}$ (dest.), $51 \mu \mathrm{lBS}$ (pH 6.5), $5 \mu$ l chymotrypsin $\left(5 \mathrm{mg} \mathrm{ml}^{-1}\right)$ and $1 \mu \mathrm{l}$ cricket haemolymph; leave the mixture for $5 \mathrm{~min}$ standing on the bench, and add $50 \mu \mathrm{l} \mathrm{L}$-dopa (18 mM in PBS). The reaction was allowed to proceed for $60 \mathrm{~min}$ in a pre-warmed $\left(30^{\circ} \mathrm{C}\right)$ micro-plate reader (Versamax, Molecular Devices, Sunnyvale, CA, USA). Softmax PRO 4.0 (Molecular Devices) software calculated the absorbency of the samples every 15 s (241 readings) and produces a kinetic curve of melanisation. Enzyme activity was determined by examining the slope of the linear phase of the reaction. As the rate during the linear phase of the reaction is proportional to the amount of enzyme present in the sample, the value given by the Softmax PRO software's autoslope function was used as the unit for the activity of enzyme concentration (Armitage and Siva-Jothy, 2005). Reactions were performed in sterile 96-well culture plates. All compounds were kept and prepared on ice and L-dopa solutions were prevented from light contact.

\section{Lysozyme assay}

Lysozyme-like activity is thought to give a constant protection against pathogens, is partly constitutive, and is involved in the lysis of bacterial cell walls (Kurtz et al., 2000). Lysozyme-like activity in the haemolymph was assessed following the protocol of Haine et al. (2007): $1 \mu \mathrm{l}$ of the thawed haemolymph was pipetted into holes ( $1 \mathrm{~mm}$ diameter), which had been punched into an agar plate containing lyophilized Micrococcus luteus bacteria. Plates were incubated at $30^{\circ} \mathrm{C}$ for $48 \mathrm{~h}$. Pictures were taken with a digital camera and clearance zones measured with the program Image-Pro Plus 5.1.

\section{Quantitative genetic analyses}

To simultaneously estimate the additive genetic basis of phenotypic variance and covariance and to test for plastic responses of each trait mean to the experimental treatment, we adopted an animal model approach. An animal model is a linear mixed-effect model in which an individual's additive genetic merit is included as a random effect (Henderson, 1976; Meyer, 1985; Shaw, 1987). Given pedigree information, this technique allows phenotypic variance to be partitioned into (additive) genetic and non-genetic components (for a review, see Kruuk, 2004). We fitted both univariate and multivariate models as described below using ASReml 2.0 (VSN International Ltd, Hemel Hempstead, UK).

Before analysis, haemocyte number and proPOV were square root transformed to normalize while lysozyme activity was rescaled $(\times 100)$ for numerical reasons (model convergence is more easily achieved with similar scaling of traits). Visual inspection of model residuals confirmed that the assumed normal error structures were appropriate for all (transformed) traits with the exception of survival after bacterial challenge (a binary trait).

\section{Univariate models}

For each trait $y$ we modelled the phenotype of individual $i$ raised in box $x$ as:

$$
y_{i}=\mu+\text { Sex }+ \text { Treatment }+ \text { Sex }: \text { Treatment }+ \text { Density }+a_{i}+b_{x}+e_{i}
$$

where $\mu$ is the phenotypic mean, Sex and Treatment (that is, early vs late photoperiod) are two-level factors. Density (number of crickets in the container at the end of experiment) is included as a fixed covariate to account for any effects on the mean caused by differing levels of mortality within boxes. Breeding values $a_{\mathrm{i}}$ are assumed to be normally distributed with zero mean and variance $\sigma_{\mathrm{A}}^{2}$, the additive genetic variance to be estimated. (Note that following popular convention we use $\sigma^{2}$ to denote the true variance component but $V$ to denote its estimate). Among individuals the variance-covariance matrix of additive effects is assumed equal to $A \sigma^{2}$ where $A$ is the additive numerator relationship matrix such that $A_{i j}=2 \Theta_{i j}$ (and $\Theta_{i j}$ is the pedigreederived coefficient of coancestry between individuals $i$ and $j$ in the pedigree). Box of rearing $\left(b_{\mathrm{x}}\right)$ was included as a random effect to reduce the potential for upward bias of genetic variance because of sharing of common environment (box) effects among sibling. Residual errors $\left(e_{i}\right)$ were assumed to be distributed as $e_{\mathrm{i}} \sim N\left(0, \sigma_{\mathrm{R}}^{2}\right)$ and uncorrelated among individuals. Trait heritabilities (with associated standard errors) were estimated as $V_{\mathrm{A}} / V_{\mathrm{P}}$ where total phenotypic variance $\left(V_{\mathrm{P}}\right)$ was calculated as the sum of all variance components). For survival after bacterial challenge, the estimate of heritability on the observed (binary) scale was transformed to an underlying liability scale following the method of Dempster and Lerner (1950).

Note that preliminary modelling of sex-specific traits (not shown) provided no statistical support for genotype-by-sex interactions (manifest as differences in $V_{\mathrm{A}}$ between the sexes and/or cross-sex genetic correlations $<1$ ). Consequently, we pooled males and females for analysis. Note that maternal effects were also tested for by inclusion of maternal identity as a random effect but were not statistically supported for any trait (likelihood ratio tests, all $P>0.05$; full results not shown). On this basis, we present parameter estimates from the model presented in equation 1. However, we acknowledge that our breeding design likely has limited power to disentangle any maternal effects that might be present from the additive genetic variance component.

\section{Among-trait (co) variance structure}

To test for genetic correlations underlying hypothesized among-trait trade-offs, we then used a multivariate formulation of Equation 1 (with all traits modelled simultaneously) to estimate among-trait covariance structure. Between each pair of traits the estimated additive genetic, common environment and residual sources of covariance (denoted $\mathrm{COV}_{\mathrm{A}}, \mathrm{COV}_{\mathrm{B}}$ and $\mathrm{COV}_{\mathrm{R}}$ ) were summed to give an estimate of the total phenotypic covariance $\left(\mathrm{COV}_{\mathrm{P}}\right)$. All covariance estimates were rescaled to their corresponding correlations. We performed a 'global' test for genetic covariance among traits by comparing the likelihood of the full multivariate model to one in which all off-diagonal elements of the genetic variance-covariance matrix (G) were constrained to zero using a likelihood ratio test. We also tested the significance of individual elements of $\mathbf{G}$ (that is, genetic covariances between specific traits) using likelihood ratio tests. Eigen decomposition was used to provide a descriptive summary of the principle axes of multivariate genetic correlation structure. Note we decompose the correlation matrix rather than $\mathbf{G}$ itself as scaling differences between the (transformed) traits are not biologically informative here.

As a complementary approach to interpreting the structure of $\mathbf{G}$ we also compared estimates of evolvabilities $\left(I_{\mathrm{A}}\right)$ and conditional evolvabilities (Hansen et al., 2003, Hansen and Houle 2008). Evolvability can be interpreted as the potential response of an individual trait to selection, while the conditional evolvability can be interpreted as the potential response if other traits in $\mathbf{G}$ were constrained to remain constant. Thus, the difference between these can provide insight into the potential for individual traits to be evolutionarily constrained by covariance with other aspects of the phenotype (Hansen et al., 2003). Evolvabilities were estimated by refitting the multivariate model after meanstandardizing (transformed) phenotypic values for all traits. We note that the interpretation of mean-standardized measures may be problematic for non-normal traits and here our estimates pertaining to transformed scales (as described above) not to traits on the observed scale. As it is not possible to appropriately transform survival after bacterial challenge (a binary trait) we do not present $I_{\mathrm{A}}$ or conditional evolvability for this trait.

\section{Genotype by environment interaction}

We then tested for genotype by environment interactions at the level of the multivariate phenotype by defining treatment specific 'sub-traits' for each of our response variables. Two multivariate animal models were fitted (as described) above to estimate a $5 \times 5 \mathrm{G}$ matrix among each set of treatmentspecific sub-traits. To see if $\mathbf{G}$ differs between the two treatments, we used a likelihood ratio tests to compare the model where $\mathbf{G}_{\text {late }}$ was freely estimated to one in which elements of $\mathbf{G}_{\text {late }}$ were fixed to the corresponding residual 
maximum likelihood estimates for the early treatment. We then applied the analogous test to $\mathbf{G}_{\text {early }}$. Although somewhat inelegant, this approach was used as we were unable to obtain stable model convergence when we attempted to include all 10 sub-traits in a single multivariate analysis. However, it should be noted that we are thus unable to formally test whether $\mathbf{G}_{\text {late }}$ differs significantly from $\mathbf{G}_{\text {early }}$ but rather test whether $\mathbf{G}_{\text {late }}$ differs significantly from the residual maximum likelihood estimate of $\mathbf{G}_{\text {early }}$ and vice versa.

We also tested for $\mathrm{G} \times \mathrm{E}$ in each response trait separately by comparing two bivariate models; one with additive genetic covariance matrix for the pair of treatment-specific sub-traits freely estimated, and a second with $V_{\mathrm{A}}$ equal in the two photoperiod treatments and $r_{\mathrm{G}}=1$ across treatments. The models were compared by likelihood ratio test as an explicit test for $\mathrm{G} \times \mathrm{E}$ for each trait.

\section{RESULTS}

Phenotypic plasticity in immune function and life history

Based on fixed effect estimates from the univariate animal models (Table 1), treatment (experimentally induced photoperiod) significantly affected cricket development time, and body size. However, the direction of the effect was contrary to our expectations and probably of minor biological relevance, as animals from the earlier photoperiod developed on average only 1-day faster than the ones from the later photoperiod, and grew to a smaller size. In contrast, none of the four components of the immune system showed any plasticity in response to the different photoperiodic environments (Table 1). Photoperiod did not affect the rate of cricket mortality after the infection with live pathogens $\left(\mathrm{F}_{1,210.5}=0.01, P=0.939\right.$, Table 1$)$, which was similar in the two treatments (early photoperiod: $41.56 \%$; late photoperiod: $43.28 \%$ ). The parameters sex and density were included in the model to statistically control for confounding influences. Both significantly affected development time (females, and individuals in boxes with higher density developed faster), and proPO (females, and individuals in boxes with lower density had a higher proPO activity), whereas sex significantly affected haemocytes and lysozyme activity (males had more haemocytes and a higher lysozyme activity), and density had a significant effect on body size (individuals in boxes with lower density grew to bigger sizes; Table 1).

\section{Quantitative genetic parameters}

Univariate animal models provided evidence of significant additive genetic variance for all traits except lysozyme activity (Table 2). There was also evidence of common environment (box) effects with $V_{\mathrm{B}}$ significantly greater than zero for all traits except lysozyme activity and proPO. Heritabilities estimated from the models ranged from $<1 \%$ (lysozyme activity) to over $70 \%$ in the case of development time. For the trait of survival after bacterial challenge, the estimated heritability ( \pm s.e.) of $0.153 \pm 0.064$ on the observed (that is, $0 / 1$ data scale) yields an estimate of $h^{2}=0.243 \pm 0.102$ on an assumed continuous 'liability' scale after applying the Dempster-Lerner transformation (Dempster and Lerner, 1950; Roff, 2001). Note that while the likelihood ratio test of $V_{\mathrm{A}}$ may not be strictly valid for this trait (because of major departures from the assumption of Gaussian residuals) the presence of nominally significant genetic variance is

Table 1 Fixed effects on all life history and immune traits

\begin{tabular}{|c|c|c|c|c|c|c|}
\hline Trait & Effect & Coefficient (s.e.) & Numerator DF & Denominator DF & Conditional F & $\mathrm{P}$ \\
\hline \multirow[t]{5}{*}{ Dev. time } & $\mu$ & $53.09(0.664)$ & 1 & 54.9 & 7125.12 & $<0.001$ \\
\hline & Sex & $0.957(0.285)$ & 1 & 955.3 & 35.33 & $<0.001$ \\
\hline & $T r t$ & $-0.874(0.365)$ & 1 & 229.6 & 4.18 & 0.043 \\
\hline & Trt.Sex & $0.511(0.404)$ & 1 & 960.4 & 1.6 & 0.207 \\
\hline & Density & $-0.517(0.127)$ & 1 & 267.7 & 16.52 & $<0.001$ \\
\hline \multirow[t]{5}{*}{ Body size } & $\mu$ & $6.869(0.054)$ & 1 & 33.2 & 19170.69 & $<0.001$ \\
\hline & Sex & $0.001(0.034)$ & 1 & 1038.9 & 1.61 & 0.205 \\
\hline & Trt & $-0.114(0.04)$ & 1 & 229.2 & 7.32 & 0.008 \\
\hline & Trt.Sex & $0.062(0.049)$ & 1 & 1030.6 & 1.61 & 0.205 \\
\hline & Density & $-0.024(0.012)$ & 1 & 261.3 & 3.96 & 0.049 \\
\hline \multirow[t]{5}{*}{ Haemocytes } & $\mu$ & $17.91(1.162)$ & 1 & 32.1 & 343.23 & $<0.001$ \\
\hline & Sex & $2.575(0.84)$ & 1 & 1016.5 & 5.95 & 0.016 \\
\hline & Trt & 0.485 (0.909) & 1 & 236.2 & 1.34 & 0.248 \\
\hline & Trt.Sex & $-2.246(1.188)$ & 1 & 1000.9 & 3.57 & 0.06 \\
\hline & density & $0.433(0.247)$ & 1 & 229.1 & 3.09 & 0.081 \\
\hline \multirow[t]{5}{*}{ ProPo } & $\mu$ & $3.541(0.101)$ & 1 & 34.1 & 1531.52 & $<0.001$ \\
\hline & Sex & $-0.081(0.074)$ & 1 & 923.5 & 4.11 & 0.044 \\
\hline & Trt & $0.043(0.078)$ & 1 & 224.2 & 0.1 & 0.755 \\
\hline & Trt.Sex & $-0.049(0.104)$ & 1 & 903.6 & 0.22 & 0.64 \\
\hline & Density & $-0.042(0.021)$ & 1 & 208.3 & 4.12 & 0.044 \\
\hline \multirow[t]{5}{*}{ Lys. activity } & $\mu$ & 48.12 (1.278) & 1 & 28.2 & 2430.25 & $<0.001$ \\
\hline & Sex & $9.172(1.01)$ & 1 & 830.2 & 171.81 & $<0.001$ \\
\hline & Trt & $-0.047(1.079)$ & 1 & 217.6 & 0 & 0.985 \\
\hline & Trt.sex & 0.107 (1.408) & 1 & 824.7 & 0.01 & 0.94 \\
\hline & Density & $0.481(0.269)$ & 1 & 195.3 & 3.21 & 0.075 \\
\hline \multirow[t]{5}{*}{ Survived } & $\mu$ & $0.566(0.07)$ & 1 & 34.6 & 82.17 & $<0.001$ \\
\hline & Sex & $-0.013(0.049)$ & 1 & 803.1 & 0.04 & 0.835 \\
\hline & Trt & $-0.019(0.052)$ & 1 & 210.5 & 0.01 & 0.939 \\
\hline & Trt.Sex & $0.041(0.068)$ & 1 & 771.9 & 0.36 & 0.549 \\
\hline & Density & $0.003(0.015)$ & 1 & 202.8 & 0.06 & 0.812 \\
\hline
\end{tabular}

Coefficients were estimated from univariate animal models with a random effect structure as described in the main text. Note that sex denotes the effect of being male or female, while treatment (Trt) denotes the effect of the early relative to the late mean. 
Table 2 Observed sample mean ( \pm s.e.), sample size $(M)$ and estimated variance components ( \pm s.e.) for all life history and immune traits

\begin{tabular}{|c|c|c|c|c|c|c|c|c|}
\hline Trait & Mean & $\mathrm{N}$ & $\mathrm{V}_{A}$ & $P$ & $\mathrm{~V}_{B}$ & $P$ & $\mathrm{~V}_{R}$ & $h^{2}$ \\
\hline \multicolumn{9}{|l|}{ Life history } \\
\hline Development time & $51.3 \pm(0.134)$ & 1084 & $14.64 \pm 3.155$ & $<0.001$ & $4.046 \pm 0.649$ & $<0.001$ & $1.234 \pm 1.639$ & $0.735 \pm 0.107$ \\
\hline \multicolumn{9}{|l|}{ Immune function } \\
\hline$\sqrt{ }$ haemocyte number & $20.6 \pm(0.301)$ & 1027 & $7.213 \pm 4.092$ & 0.026 & $5.855 \pm 3.065$ & 0.035 & $78.99 \pm 4.790$ & $0.078 \pm 0.044$ \\
\hline Survived after bacterial challenge & $0.572 \pm(0.017)$ & 811 & $0.039 \pm 0.0166$ & 0.002 & $0.021 \pm 0.010$ & 0.020 & $0.189 \pm 0.015$ & $0.153 \pm 0.064$ \\
\hline
\end{tabular}

$V_{B}$ denotes variance because of box effects and $V_{R}$ is residual variance and $V_{A}$ is the additive genetic variance. $P$-values denote the significance of $V_{A}$ or $V_{B}$ based on comparison of the full model to a reduced model with no additive or box effect as appropriate (likelihood ratio tests on one degree of freedom). Heritabilities $\left(h^{2} \pm \mathrm{s}\right.$.e.) are estimated as the ratio of additive variance $\left(V_{A}\right)$ to total phenotypic variance (determined as the sum of all variance components).

Table 3 Variance covariance matrices and correlations ( \pm s.e.) for development time, body size, haemocyte number, proPO and survival after bacterial challenge

\begin{tabular}{|c|c|c|c|c|c|}
\hline \multicolumn{6}{|l|}{ Residual } \\
\hline Dev. time & $1.317 \pm 1.626$ & $0.899 \pm 0.583$ & $-0.277 \pm 0.152$ & $-0.248 \pm 0.218$ & $0.001 \pm 0.203$ \\
\hline Haemocytes & $-2.779 \pm 1.853$ & $-0.488 \pm 0.148$ & $76.751 \pm 4.776$ & $0.284 \pm 0.043$ & $0.028 \pm 0.051$ \\
\hline ProPO & $-0.213 \pm 0.149$ & $-0.018 \pm 0.013$ & $1.862 \pm 0.312$ & $0.562 \pm 0.038$ & $0.112 \pm 0.054$ \\
\hline Survived & $0.001 \pm 0.057$ & $-0.005 \pm 0.009$ & $0.107 \pm 0.197$ & $0.036 \pm 0.018$ & $0.188 \pm 0.015$ \\
\hline Dev. time & $14.470 \pm 3.131$ & $-0.174 \pm 0.222$ & $-0.824 \pm 0.132$ & $-0.123 \pm 0.223$ & $0.019 \pm 0.231$ \\
\hline Body size & $-0.105 \pm 0.133$ & $0.025 \pm 0.011$ & $0.491 \pm 0.328$ & $-0.111 \pm 0.306$ & $0.536 \pm 0.279$ \\
\hline Haemocytes & $-9.701 \pm 3.089$ & $0.242 \pm 0.156$ & $9.593 \pm 4.400$ & $0.301 \pm 0.293$ & $0.042 \pm 0.325$ \\
\hline ProPO & $-0.131 \pm 0.242$ & $-0.005 \pm 0.014$ & $0.261 \pm 0.287$ & $0.079 \pm 0.035$ & $0.222 \pm 0.316$ \\
\hline Survived & $0.013 \pm 0.168$ & $0.016 \pm 0.010$ & $0.025 \pm 0.190$ & $0.012 \pm 0.017$ & $0.035 \pm 0.016$ \\
\hline ProPO & $0.057 \pm 0.084$ & $-0.008 \pm 0.009$ & $0.042 \pm 0.181$ & $0.013 \pm 0.021$ & $-0.058 \pm 0.588$ \\
\hline Survived & $-0.066 \pm 0.060$ & $0.002 \pm 0.006$ & $0.338 \pm 0.128$ & $-0.001 \pm 0.011$ & $0.024 \pm 0.010$ \\
\hline \multicolumn{6}{|l|}{ Phenotypic } \\
\hline Dev. time & $19.87 \pm 1.615$ & $0.149 \pm 0.041$ & $-0.354 \pm 0.031$ & $-0.080 \pm 0.039$ & $-0.023 \pm 0.044$ \\
\hline Body size & $0.28 \pm 0.077$ & $0.178 \pm 0.009$ & $-0.105 \pm 0.033$ & $-0.091 \pm 0.034$ & $0.012 \pm 0.008$ \\
\hline Haemocytes & $-15.18 \pm 1.802$ & $-0.425 \pm 0.137$ & $92.69 \pm 4.271$ & $0.278 \pm 0.032$ & $0.469 \pm 0.177$ \\
\hline ProPO & $-0.287 \pm 0.141$ & $-0.031 \pm 0.012$ & $2.164 \pm 0.274$ & $0.653 \pm 0.031$ & $0.047 \pm 0.016$ \\
\hline Survived & $-0.052 \pm 0.097$ & $0.059 \pm 0.039$ & $0.098 \pm 0.036$ & $0.117 \pm 0.038$ & $0.248 \pm 0.013$ \\
\hline
\end{tabular}

All estimates were obtained from a single (five trait) multivariate model. Variance components are on the diagonal with covariances below and correlations above. Bold denotes a value nominally significant at the $5 \%$ level based on estimated standard errors.

supported by the estimated standard errors for $V_{\mathrm{A}}$ and $h^{2}$ (on either scale).

Multivariate analysis of all traits simultaneously yielded quantitatively similar estimates of trait variance components as well as estimates of the covariance/correlation structures among traits (Table 3). Note that inclusion of $100^{\star}$ lysozyme activity in the analysis caused problems for model stability and estimation of the $\mathbf{G}$ matrix, presumably because of the effective absence of detectable $V_{\mathrm{A}}$ (as shown by univariate models). We therefore excluded this trait. Based on the estimated standard errors, phenotypic correlations between most pairs of traits were statistically significant although for the most part effect sizes were generally small to moderate. Phenotypic correlations were positive within the trait types (that is, life history and immune) but all significant correlations between life history and immune traits were negative.

We found strong negative genetic correlation $\left(r_{\mathrm{G}}=-0.824 \pm 0.132\right)$

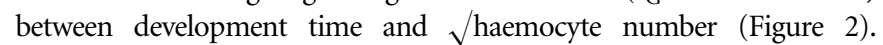
Significance of this estimate (suggested by the associated standard error) was formally tested by likelihood ratio test (comparison of bivariate animal models: $r_{\mathrm{G}}$ unconstrained $v \mathrm{r}_{\mathrm{G}}$ fixed at zero, $\left.\chi_{1}^{2}=14.9, P<0.001\right)$. Likelihood ratio tests were similarly used to confirm the significance of common environment (box) correlations 


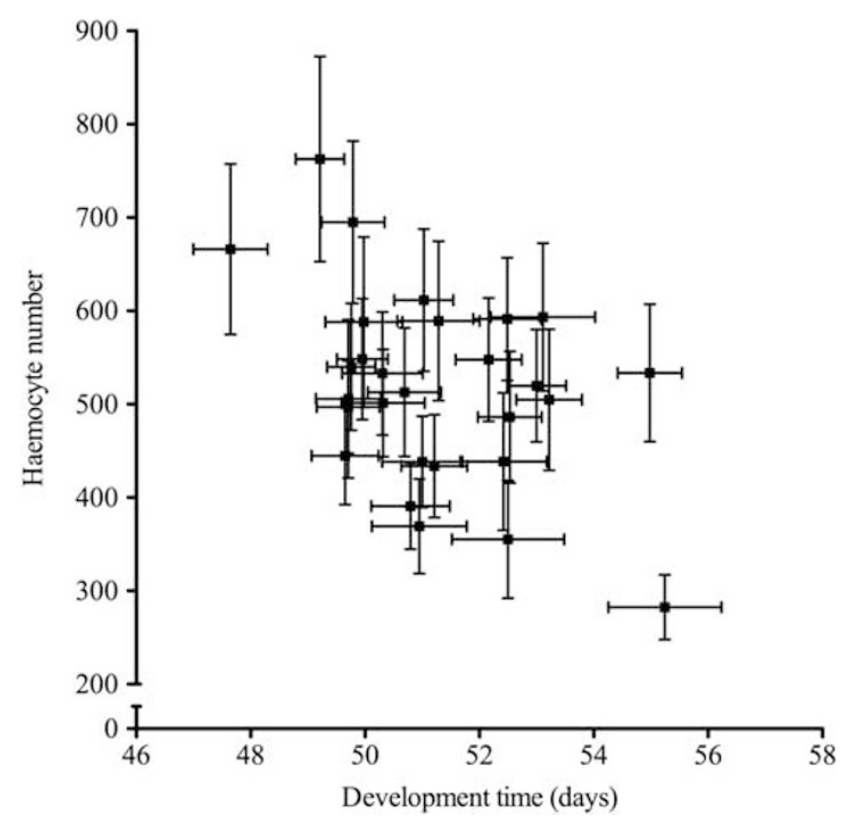

Figure 2 Negative genetic correlation between development time and haemocyte numbers in G. bimaculatus. Shown are means per sire ( \pm s.e.; $N=28$ ).

between development time and $\sqrt{\text { haemocyte number }}\left(r_{\mathrm{Box}}=\right.$ $\left.-0.529 \pm 0.173, \chi_{1}^{2}=6.54, P=0.011\right)$, and between $\sqrt{ }$ haemocyte number and survival after bacterial challenge $\left(r_{\mathrm{Box}}=0.857 \pm 0.321\right.$ from multivariate model; comparison of bivariate models $\chi_{1}^{2}=6.88, P=0.009$ ).

Despite the statistical uncertainty around individual estimates of genetic (and other) correlations, there was nonetheless evidence to support the presence of genetic covariance among traits in $\mathbf{G}$ overall. Thus, the full multivariate model is a significantly better fit to the data than one in which $\mathbf{G}$ contains no off-diagonal elements (that is, $\mathrm{COV}_{\mathrm{A}}=0$ for all trait pairs; $\chi^{2}{ }_{10}=23.4, P=0.009$ ).

Eigenvector decomposition of the genetic correlation matrix revealed that the first eigenvector explains $43 \%$ of the variance with loadings that are antagonistic with respect to development time and all other traits. However, the importance of this pattern should not be overstated given that the second and third eigenvectors also accounted for substantial variance at $27 \%$ and $21 \%$, respectively. Nevertheless, it is notable that this axis of variation actually runs counter to our a priori prediction of a genetic trade-off between development time and immune function.

Evolvabilities and conditional evolvabilities for all traits (except for survival after bacterial challenge) were estimated (Figure 3). Evolvabilities were $<1 \%$ (except for number of haemocytes), indicating that potential evolutionary responses are $<1 \%$ per generation. These values are upper limits as when conditioning for the other traits in $\mathbf{G}$, the conditional evolvabilities are even lower (Figure 3).

Analysis of treatment-specific sub-traits provided no evidence of $\mathrm{G} \times \mathrm{E}$ and treatment-specific estimates of $\mathbf{G}$ were similar (not shown). The multivariate model fit for traits measured under late photoperiod conditions was not significantly worsened by fixing $\mathbf{G}$ at the parameter values estimated using the data from the early treatments $\left(\chi^{2}{ }_{15}=9.34\right.$, $P=0.859)$. Similarly, the model fit to early data were not significantly reduced by constraining $\mathbf{G}$ to parameter estimates obtained from the late treatment $\left(\chi^{2}{ }_{15}=0.16, P=0.859\right)$. Bivariate models testing on a trait by trait basis also provided no evidence for $\mathrm{G} \times \mathrm{E}$ effects on any of the immune or life history response variables measured (Table 4).

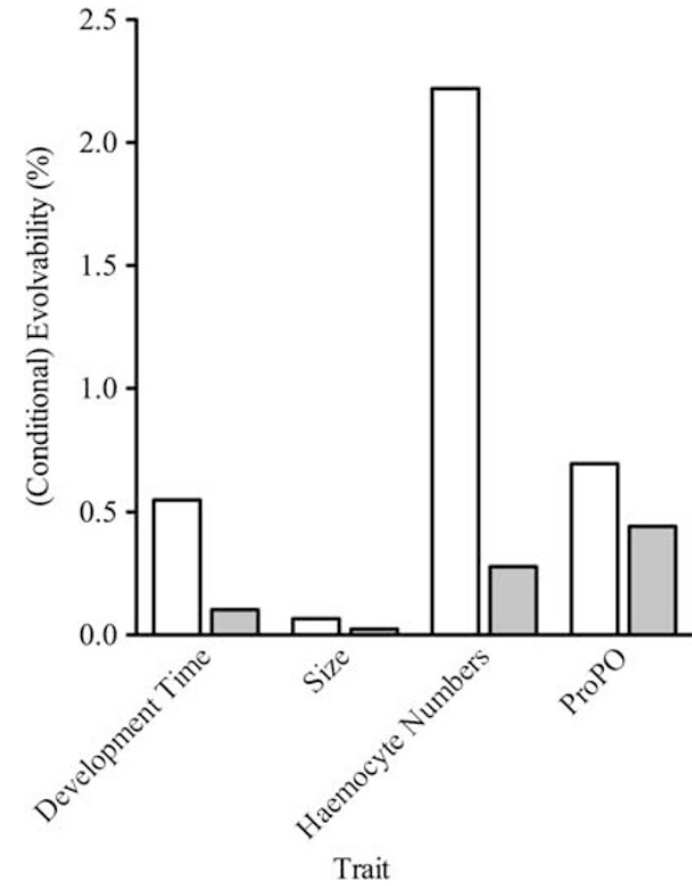

Figure 3 Evolvabilities (\%, light bars) and conditional evolvabilities (\%, grey bars) for all traits, except for survival after bacterial challenge.

Table 4 Tests for $\mathbf{G} \times \mathbf{E}$

\begin{tabular}{lcclc}
\hline Trait & G $\times E$ model & Constrained model & $\chi^{2} 2$ & $P$ \\
\hline Dev. time & -1933.63 & -1933.84 & 0.42 & 0.8106 \\
Body size & 421.056 & 420.503 & 1.106 & 0.5752 \\
Haemocytes & -2829.16 & -2829.95 & 1.58 & 0.4538 \\
ProPO & -263.444 & -266.064 & 5.24 & 0.0728 \\
Survived & 165.003 & 164.702 & 0.602 & 0.7401
\end{tabular}

For each trait assayed a $\mathrm{G} \times \mathrm{E}$ model ( $V_{\mathrm{A}}$ free to vary between treatments, $-1 \leqslant r_{\mathrm{G}} \leqslant+1$ across treatments) was compared with a constrained model (no $G \times E$ allowed, $V_{A}$ equal across treatments, $r_{G}=+1$ ) by likelihood ratio test. Twice the difference in log-likelihoods is assumed to be distributed as $\chi^{2}$ with two degrees of freedom.

\section{DISCUSSION}

Photoperiod affected age and size at maturity, but in a direction contrary to theoretical predictions (Rowe and Ludwig, 1991; Abrams et al., 1996) and empirical findings in other insect species (De Block and Stoks, 2003, 2004). Crickets kept under late season photoperiod conditions, that is, decreasing day length, did not accelerate their development, but took 1-day longer, and concurrently grew to bigger sizes as adults compared with the ones raised under early photoperiod conditions (resulting in no growth rate differences between the two treatments). None of the measured immune parameters was significantly affected by photoperiod, indicating low phenotypic plasticity for these traits under these conditions.

Quantitative genetic analysis revealed significant additive genetic variance for all life history and immunity traits, except for lysozyme activity (also reported for Gryllus firmus by Rantala and Roff, 2006). We found mostly positive phenotypic correlations within immune traits and within life history traits, and mostly negative phenotypic correlations between immune and life history traits. There was, however, little support for significant genetic correlations between traits, apart from the negative genetic correlation between develop- 
ment time and haemocyte number. The photoperiod did not affect the sign or magnitude of genetic correlations.

Although in most other studies insects accelerated their development when time-constrained (De Block and Stoks, 2003, 2004), Rolff et al. (2004) found this only for time-constrained damselflies, which were additionally food-constrained. Most probably all larvae kept at high food level had already attained their minimum development time so that any further acceleration was not possible (Rolff et al., 2004). In our experiment, food was available ad libitum and, with an average development time of 50 and 51 days (early vs late photoperiod, respectively), crickets developed as fast as G. bimaculatus individuals in other rearing experiments under similar conditions (Bigelow, 1962; Merkel, 1977; Behrens et al., 1983; Rodriguez-Muñoz et al., 2008; Ibler et al., 2009). It is thus possible that the crickets in our experiment had, in both treatments, reached the minimum development time for that set of environmental conditions (access food, temperature $26^{\circ} \mathrm{C}$ ), although faster development of just under 30 days is physiologically feasible under higher temperatures (Behrens et al., 1983). G. bimaculatus are considered to be multivoltine and to develop without a diapause. The late photoperiod individuals took on average 1-day longer to reach maturity, but attained a bigger size at maturity. This result is consistent with the notion of a change of voltinism along an environmental gradient, reflected in a saw tooth pattern of development and hence body size (Masaki and Walker, 1987).

The quantitative genetics analysis provided evidence of significant additive genetic variance for all traits except for lysozyme activity. Heritability estimates were all significantly different from zero (apart from lysozyme activity), and ranged from $<0.1$ (haemocyte number) to over 0.7 in the case of development time. Such a relatively large estimate for development time, a parameter closely connected to fitness, has been found in other insect species $(0.82$ for $S$. littoralis in Cotter et al., 2004) but is difficult to explain.

Cricket development time across both treatments ranged from 43 to 71 days, providing variation to investigate the effects of fast development, particularly whether fast developers suffer from an impaired immune system. We found a significant positive phenotypic correlation between development time and body size: faster developers reached adulthood at a smaller size, confirming findings of Rantala and Roff (2005) in the same species. All phenotypic correlations between life history and immune traits were negative. Individuals, which developed faster, grew to a smaller size, but had more haemocytes, and an increased proPO activity. The positive association between the two immune parameters confirm previous findings by Cotter et al. (2004), who found a significant positive (genetic) correlation between haemocyte density and PO activity in the haemolymph in Spodoptera littoralis. Most importantly, the positive correlation in our study between both immune parameters and survival after the $\mathrm{LD}_{50}$ dose of Serratia marcescens shows that individuals with more haemocytes and proPO activity, are more likely to survive. This indicates that the measured immune parameters can be taken as a direct measure of resistance against this pathogen (for details, see Adamo, 2004).

We detected a strong negative genetic correlation between development time and haemocyte density $\left(r_{\mathrm{G}}=-0.824 \pm 0.132\right)$. This is contrary to our a priori expectation that immunity and development time are traded off. Instead we found that genes predisposing to fast growth are also associated with greater investment into immunity, thereby increasing resistance to pathogens. So far, this has never been found in any other study on invertebrates (Cotter et al., 2004; Rolff et al., 2005), but in a wild population in Soay sheep, where genetically resistant individuals showed superior growth (Coltman et al., 2001). Negative correlations between development time and life history traits have been reported in a number of species (Roff, 2000), especially in insects. At face value such correlations are contrary to predictions from life history, as they would create a superorganism (Reznick et al., 2000): an organism that not only develops fast but also has, for example, higher fecundity than individuals developing more slowly. However, it is important to acknowledge that the bivariate view of traits involved in trade-offs may often be overly simplistic, such that scrutinizing pairwise genetic correlations will be inadequate to infer evolutionary constraint (Walsh and Blows, 2009). For example, Reznick et al. (2000) suggested that positive (with respect to fitness consequences) genetic correlations between life history traits could be driven by genetic variation for resource acquisition. We do not have a direct answer for the fact that we found fast developing crickets to have higher haemocyte densities (and hence exhibiting higher resistance against an entomopathogen). It has been shown in Drosophila melanogaster that individuals selected for parasitoid resistance, mediated by increased haemocyte densities, suffer costs under low resource availability (Kraaijeveld and Godfray, 1997). Fellowes et al. (1999) showed that this cost was caused by a reduction in feeding rate and speculated that the underlying developmental pathway leads to the same embryo tissues giving rise to jaw muscles and the haemotopoietic organ. Whether similar mechanisms apply to the crickets remains to be explored.

Although we found phenotypic responses to the experimental manipulation of the photoperiod, there was no support for $\mathrm{G} \times \mathrm{E}$ in any of the traits assayed. Consequently, the sign and magnitude of among-trait correlations will not differ between the two environments. This finding is in contrast to other studies reporting changes in genetic correlations in different environments (reviewed in Sgrò and Hoffmann, 2004), although only one study manipulated the photoperiod (Giesel, 1986). Giesel (1986) reported changes in the sign of genetic correlations between life history traits in Drosophila melanogaster. We are not aware of any quantitative genetic studies that also estimated physiological, and especially immunological, parameters in different environments. G. bimaculatus is multivoltine and therefore the breeding season and the photoperiodic changes experienced are variable. It is therefore possible that life history traits are relatively plastic (development time varied by a factor of 1.7) in this species and are hence not affected. Immune traits are phenotypically correlated and physiologically integrated. This integration could impose a constraint that prevents the environmental disruption of genetic correlations.

Moreover, when estimating conditional evolvabilities, that is, assuming that all other traits in our study were under stabilizing selection, the potential for autonomous evolution of single traits was rather limited. Given the similar quantitative genetic architecture in both environments, one would predict very similar evolutionary trajectories for the traits measured. Although selection can act independently on life history traits and immune traits, our data suggest that in crickets in our two experimental environments the potential for independent evolution of those traits is very limited.

In short, the evolvability of resistance against bacterial pathogens, because of the broad-spectrum nature of the insect immune system (Rolff and Reynolds, 2009), is the same in both environments. An important antibacterial response, the encapsulation response, for example requires the co-ordinated action of PO and haemocytes. It is noteworthy that Simons and Roff (1996) observed very similar genetic correlations for morphological traits, much less so for life history traits, in two different environments (laboratory vs field) in the cricket Gryllus pennsylvanicus. Although $\mathrm{G} \times \mathrm{E}$ effects have been commonly observed, we find no support for across-treatment changes in genetic correlations here. 


\section{DATA ARCHIVING}

Data have been deposited at Dryad: doi:10.5061/dryad.hm06n7c0.

\section{CONFLICT OF INTEREST}

The authors declare no conflict of interest.

\section{ACKNOWLEDGEMENTS}

We thank T Hansen for discussion, D Mikolajewski, S Parratt, A Plischke, E Harney and R Naylor for their assistance in the laboratory, and K Reinhardt and three anonymous referees for valuable comments. KH was supported by the DAAD (German Academic Exchange Service), PD by the Alexander von Humboldt Foundation, and AJW by NERC (Natural Environment Research Council) and the BBSRC (Biotechnology and Biological Sciences Research Council).

Abrams PA, Leimar O, Nylin S, Wiklund C (1996). The effect of flexible growth rates on optimal sizes and development times in a seasonal environment. Am Nat 147: 381-395.

Adamo SA (2004). Estimating disease resistance in insects: phenoloxidase and lysozymelike activity and disease resistance in the cricket Gryllus texensis. J Insect Physiol 50: 209-216.

Armitage SAO, Siva-Jothy MT (2005). Immune function responds to selection for cuticular colour in Tenebrio molitor. Heredity 94: 650-656.

Barnes Al, Siva-Jothy MT (2000). Density-dependent prophylaxis in the mealworm beetle Tenebrio molitor L- (Coleoptera: Tenebrionidae): cuticular melanization is an indicator of investment in immunity. Proc $R$ Soc Lond Ser B-Biol Sci 267: 177-182.

Behrens W, Hoffmann KH, Kempa S, Gassler S, Merkelwallner G (1983). Effects of diurnal thermoperiods and quickly oscillating temperatures on the development and reproduction of crickets Gryllus bimaculatus. Oecologia 59: 279-287.

Bigelow RS (1962). Factors affecting developmental rates and diapause in field crickets. Evolution 16: 396-406

Bradshaw W, Holzapfel CM (1996). Genetic constraints to life-history evolution in the pitcher-plant mosquito, Wyeomyia smithii. Evolution 50: 1176-1181.

Chippindale AK, Hoang DT, Service PM, Rose MR (1994). The evolution of development in Drosophila melanogaster selected for postponed senescence. Evolution 48: 1880-1899.

Coltman DW, Pilkington J, Kruuk LEB, Wilson K, Pemberton JM (2001). Positive genetic correlation between parasite resistance and body size in a free-living ungulate population. Evolution 55: 2116-2125.

Cotter SC, Kruuk LEB, Wilson K (2004). Costs of resistance: genetic correlations and potential trade-offs in an insect immune system. J Evolution Biol 17: 421-429.

De Block M, Stoks R (2003). Adaptive sex-specific life history plasticity to temperature and photoperiod in a damselfly. J Evolution Biol 16: 986-995.

De Block M, Stoks R (2004). Life-history variation in relation to time constraints in a damselfly. Oecologia 140: 68-75.

De Block M, Stoks R (2005). Fitness effects from egg to reproduction: bridging the life history transition. Ecology 86: 185-197.

Dempster ER, Lerner IM (1950). Heritability of threshold characters. Genetics 35 : 212-236.

Fellowes MDE, Kraaijeveld AR, Godfray HCJ (1999). The relative fitness of Drosophila melanogaster (Diptera, Drosophilidae) that have successfully defended themselves against the parasitoid Asobara tabida (Hymenoptera, Braconidae). J Evolution Biol 12. $123-128$.

Giesel JT (1986). Genetic correlation structure of life history variables in outbred, wild Drosophila melanogaster- effects of photoperiod regimen. Am Nat 128: 593-603.

Gillespie JP, Burnett C, Charnley AK (2000). The immune response of the desert locust Schistocerca gregaria during mycosis of the entomopathogenic fungus, Metarhizium anisopliae var acridum. J Insect Physiol 46: 429-437.

Gotthard K (2001). Growth strategies of ectothermic animals in temperate environments. In: Atkinson D, Thorndyke M (eds). Environment and Animal Development. Bios Scientific: Oxford, pp 287-303.

Haine ER, Rolff J, Siva-Jothy MT (2007). Functional consequences of blood clotting in insects. Dev Comp Immunol 31: 456-464.

Hansen TF, Armbruster WS, Carlson ML, Pélabon C (2003). Evolvability and genetic constraint in Dalechampia blossoms: Genetic correlations and conditional evolvability. J Exp Zool Part B 296B: 23-39.

Hansen TF, Houle D (2008). Measuring and comparing evolvability and constraint in multivariate characters. J Evolution Biol 21: 1201-1219.

Henderson CR (1976). A simple method for computing the inverse of a numerator relationship matrix used in prediction of breeding values. Biometrics 32: 69-83.

Henderson PJF (1992). Statistical analysis of enzyme kinetic data. In: Eisenthal R, Danson MJ (eds). Enzyme Assays: A Practical Approach. Oxford University Press: Oxford, pp 277-316.

Ibler B, Makert GR, Matthias WL (2009). Larval and adult development and organisation of a systemic breeding of the mediterranean field cricket (Gryllus bimaculatus de Geer, 1773). Zool Garten NF 78: 81-101.
Jacot A, Scheuber H, Kurtz J, Brinkhof MWG (2005). Juvenile immune system activation induces a costly upregulation of adult immunity in field crickets Gryllus campestris. Proc R Soc Lond Ser B-Biol Sci 272: 63-69.

Kraaijeveld AR, Godfray HCJ (1997). Trade-off between parasitoid resistance and larval competitive ability in Drosophila melanogaster. Nature 389: 278-280.

Krieg A (1987). Diseases caused by bacteria and other prokaryotes. In: Fuxa JR, Tanada $Y$ (eds). Epizootiology of Insect Diseases. Wiley and Sons: New York, pp 323-355.

Kruuk LEB (2004). Estimating genetic parameters in natural populations using the 'animal model'. Phil Trans R Soc Lond Ser B-Biol Sci 359: 873-890.

Kurtz J, Wiesner A, Götz P, Sauer KP (2000). Gender differences and individual variation in the immune system of the scorpionfly Panorpa vulgaris (Insecta: Mecoptera). Dev Comp Immunol 24: 1-12.

Ludwig D, Rowe L (1990). Life-history strategies for energy gain and predator avoidance under time constraints. Am Nat 135: 686-707.

Masaki S, Walker TJ (1987). Cricket life-cycles. Evol Biol 21: 349-423.

Merkel G (1977). The effects of temperature and food quality on the larval development of Gryllus bimaculatus (Orthoptera, Gryllidae). Oecologia 30: 129-140.

Meyer K (1985). Maximum-likelihood estimation of variance-components for a multivariate mixed model with equal design matrices. Biometrics 41: 153-165.

Nigam Y, Maudlin I, Welburn S, Ratcliffe NA (1997). Detection of phenoloxidase activity in the hemolymph of tsetse flies, refractory and susceptible to infection with Trypanosoma brucei rhodesiense. J Invertebr Pathol 69: 279-281.

Plaistow SJ, Lapsley CT, Beckerman AP, Benton TG (2004). Age and size at maturity: sex, environmental variability and developmental thresholds. Proc $R$ Soc Lond Ser B-Biol Sci 271: 919-924.

Rantala MJ, Roff DA (2005). An analysis of trade-offs in immune function, body size and development time in the mediterranean field cricket, Gryllus bimaculatus. Funct Ecol 19: 323-330.

Rantala MJ, Roff DA (2006). Analysis of the importance of genotypic variation, metabolic rate, morphology, sex and development time on immune function in the cricket, Gryllus firmus. J Evolution Biol 19: 834-843.

Reznick D, Nunney L, Tessier A (2000). Big houses, big cars, superfleas and the costs of reproduction. Trends Ecol Evol 15: 421-425.

Rodriguez-Muñoz R, Bretman A, Hadfield JD, Tregenza T (2008). Sexual selection in the cricket Gryllus bimaculatus: no good genes? Genetica 134: 129-136.

Roff D (1980). Optimizing development time in a seasonal environment-the ups and downs of clinal variation. Oecologia 45: 202-208.

Roff DA (1992). The Evolution of Life Histories. Chapman and Hall: New York.

Roff DA (2000). Trade-offs between growth and reproduction: an analysis of the quantitative genetic evidence. J Evolution Biol 13: 434-445.

Roff DA (2001). The threshold model as a general purpose normalizing transformation. Heredity 86: 404-411.

Rolff J, Armitage SAO, Coltman DW (2005). Genetic constraints, sexual dimorphism and immune defence. Evolution 59: 1844-1850.

Rolff J, Reynolds SE (2009). Insect Infection and Immunity: Evolution, Ecology and Mechanisms. Oxford University Press: Oxford.

Rolff J, Siva-Jothy MT (2003). Ecological immunology: an invertebrate perspective. Science 301: 472-475.

Rolff J, Van de Meutter F, Stoks R (2004). Time constraints decouple age and size at maturity and physiological traits. Am Nat 164: 559-565.

Rowe L, Ludwig D (1991). Size and timing of metamorphosis in complex life-cycles-time constraints and variation. Ecology 72: 413-427.

Schmid-Hempel $P$ (2003). Variation in immune defence as a question of evolutionary ecology. Proc R Soc Lond Ser B-Biol Sci 270: 357-366.

Semlitsch RD (1993). Adaptive genetic variation in growth and development of tadpoles of the hybridogenetic Rana esculenta complex. Evolution 47: 1805-1818.

Sgrò CM, Hoffmann AA (2004). Genetic correlations, tradeoffs and environmental variation. Heredity 93: 241-248.

Sharpe ES, Detroy RW (1979). Susceptibility of japanese beetle larvae to Bacillus thuringiensis - associated effects of diapause, midgut $\mathrm{PH}$, and milky disease. J Invertebr Pathol 34: 90-91.

Shaw RG (1987). Maximum-likelihood approaches applied to quantitative genetics of natural populations. Evolution 41: 812-826.

Simons AM, Roff DA (1996). The effect of a variable environment on the genetic correlation structure in a field cricket. Evolution 50: 267-275.

Siva-Jothy MT (2000). A mechanistic link between parasite resistance and expression of a sexually selected trait in a damselfly. Proc $R$ Soc Lond Ser B-Biol Sci 267: 2523-2527.

Stearns SC (1992). The Evolution of Life Histories. Oxford University Press: Oxford.

Stoks R, De Block M, Slos S, Van Doorslaer W, Rolff J (2006). Time constraints mediate predator-induced plasticity in immune function, condition, and life history. Ecology 87 : 809-815.

Thompson JJW, Armitage SAO, Siva-Jothy MT (2002). Cuticular colour change after imaginal eclosion is time-constrained: blacker beetles darken faster. Physiol Entomol 27: 136-141.

Walsh B, Blows MW (2009). Abundant genetic variation + strong selection = multivariate genetic constraints. A geometric view of adaptation. Ann Rev Ecol Evol Syst 40: 41-59.

Werner EE (1986). Amphibian metamorphosis-growth-rate, predation risk, and the optimal size at transformation. Am Nat 128: 319-341.

Zera AJ, Harshman LG (2001). The physiology of life history trade-offs in animals. Ann Rev Ecol System 32: 95-126. 